\title{
Evaluation of NS1 Antigen Detection for Early Diagnosis of Dengue Virus Infection in a Tertiary Care Hospital in Karnataka, India
}

\author{
Rajani Ranganath $^{1 *}$ and Basavaraj V. Peerapur ${ }^{2}$ \\ Department of Microbiology, Raichur Institute of Medical Sciences, Raichur, Karnataka, India \\ *Corresponding author
}

\begin{tabular}{|c|c|}
\hline & $\mathbf{R A} \mathbf{A} \mathbf{T}$ \\
\hline $\begin{array}{l}\mathrm{N} \\
\mathrm{Mc} \\
\mathrm{co}\end{array}$ & \multirow{3}{*}{$\begin{array}{l}\text { To evaluate the efficacy of NS1 antigen as a early marker for diagnosis of Dengue } \\
\text { virus infection. Performance of NS1 antigen assay in comparison to MAC ELISA } \\
\text { was evaluated when performed together in a single sample in Group } 1.391 \\
\text { acute/early convalescent sera were screened by both the assays. Group } 2 \text { evaluated } \\
\text { the specificity of NS1 assay in comparison to MAC ELISA on } 30 \text { samples included } \\
\text { as controls. In Group } 1,162(41.43 \%) \text { were positive by NS } 1 \text { antigen assay and } \\
71(18.15 \%) \text { were positive by MAC ELISA. NS } 1 \text { antigen positivity decreased from } \\
96.91 \% \text { in acute phase sera to } 3.08 \% \text { in early convalescent sera }(P<0.0001) \text {. } \\
\text { Conversely IgM positivity increased from } 7.04 \% \text { in acute phase sera to } 92.95 \% \text { in } \\
\text { early convalescent sera }(P<0.0001) \text {. All the samples in Group } 2 \text { were negative } \\
\text { showing } 100 \% \text { specificity of both the assays. NS1 Ag is an effective tool for the } \\
\text { diagnosis of DENV infection, especially with in first } 7 \text { days of illness. Combined } \\
\text { use of NS1 antigen assay with MAC ELISA test could significantly improve } \\
\text { diagnostic sensitivity of dengue infection. }\end{array}$} \\
\hline & \\
\hline & \\
\hline
\end{tabular}

\section{Introduction}

Dengue has become a major global public health problem in the developing countries. The estimated risk of acquiring Dengue virus infection (DV) is approximately 2.5 billion people living mainly in urban areas (Halstead et al., 2007). DV causes various clinical symptoms ranging from asymptomatic or undifferentiated fever, known as dengue fever (DF), dengue hemorrhagic fever (DHF), dengue shock syndrome (DSS), leading to death, especially among children (Gubler et al., 1999).
Viral isolation by culture or viral RNA detection by PCR helps in the diagnosis of recent dengue infection. But this viral isolation is very time consuming and requires specialized laboratory equipment (Sathish et al., 2003). Newer PCR types like nested reverse transcription-PCR (RT-PCR) and realtime RT-PCR have significantly reduced processing times but are expensive and technically exacting (Bessof et al., 2008). As a result, dengue culture and PCR have limited utility in routine clinical use. 
Rapid newer test for presumptive diagnosis of dengue is detection of NS1 (Non structural protein) Ag. This antigen is a highly conserved glycoprotein that is essential for the viability of dengue virus (Dussart et al., 2006). The first immunoglobulin isotype to appear is $\operatorname{IgM}$ antibody, suggesting recent infection. One of the most recent advances for routine dengue diagnosis is $\operatorname{IgM}$ antibody capture ELISA (MAC ELISA) (Vaughn et al., 2000). To confirm dengue during both early and late infection, combined usage of NS1 antigen and IgM antibody ELISA are promising (Andries et al., 2012).

Here, in this study, we have performed both types of immunoassays; NS1 ELISA and IgM ELISA, in the samples received in our laboratory and the results of the combined tests have been compared individually with each test separately.

\section{Materials and Methods}

The comparative study was conducted in Government hospital as a part of Integrated Disease Surveillance Programme (IDSP) in Raichur, Karnataka from January 2015 to December 2015. Demographic details of the patients were collected. Depending on the reporting time of the patients, sera comprised of both acute and early convalescent phases. Samples were divided into two Groups ie Group 1 and Group 2.

Group 1 was the test group which included all patients (both adults and children) with fever fitting into WHO revised classification of dengue (WHO, 2009). The samples were screened for the presence of dengue-specific IgM antibody capture enzyme linked immunsorbent assay(MAC-ELISA),using a kit prepared by the National Institute of virology, Pune, India, strictly following the manufacurer's protocol (Cecilia et al.,2011).
NS1 Ag was detected in the sera by PanBio NS1Ag ELISA kit. Data was statistically analyzed by calculating the ' $p$ ' value.

Group 2 was the control group which included 30 patients. 20 were obtained from patients with fever due to known etiology other than dengue [enteric fever (12), bacterial meningitis (3), UTI (5)] and 10 were from healthy blood donors. All these 30 were screened by both the assays.

\section{Results and Discussion}

Out of the 391 samples in Group 1, 162 $(41.43 \%)$ samples were positive for NS1 Ag and $71(18.15 \%)$ were positive for $\operatorname{IgM}$ antibody including those that were positive by both (Table 1$)$.

157 were from acute phase sera and 5 were from early convalescent phase out of 162 NS1Ag positive samples. The NS1 Ag detection rate decreased from $96.91 \%$ in acute phase sera to $3.08 \%$ in early convalescent sera $(P<0.0001)$. All the 122 samples that were positive for NS1 Ag alone in this study group I belonged to acute phase sera (Table 2).

66 were from early convalescent phase and only 10 were from acute phase sera out of 71 MAC ELISA positive samples. All the 31 samples that were only IgM positive belonged to early convalescent phase (Table 2). $\operatorname{IgM}$ antibody detection rate increased from $7.04 \%$ to $92.95 \%(P<0.0001)$.

Total number of male patients was 210 (53.70) and female patients were 181 $(46.29 \%)$. Out of 391, highest were from 1$10 \mathrm{yr}$ age group, followed by 11-20 yrs and lowest were $>61$ yr age group (Fig 1).

Fig 2 depicts details of both IgM negative \& NS1 negative patients. 
Serum samples which were positive by both NS1 antigen ELISA \& IgM ELISA were also highest in the age group of 1-10 yrs. (Fig :3).

Fig 4 shows serum samples that were positive only by IgM.

The IgM capture ELISA is most commonly used in India due to its low cost and ease of handling. But here it is important to understand that, NS1 antigen detection assay has an advantage over IgM detection that it can diagnose a case of dengue while the latter cannot, because $\operatorname{IgM}$ and $\operatorname{IgG}$ antibodies remain detectable for months after the clinical illness and hence test results obtained from single sera are only suggestive of infection (Andries et al., 2012). To confirm a case of acute dengue infection by serology, IgM seroconversion or a fourfold increase of $\mathrm{IgG}$ antibody titer in paired sera must be demonstrated (Tricou et al.,2010).

In the present study, $31.20 \%$ sera were positive only for NSlantigen, by ELISA.
Several studies have reported a lesser values of $23.3 \%$ (Datta et al.,2010), 30\% (Kulkarni et al., 2011) as well as higher value of even $60 \%$ (Santosh Tathe et al., 2013) positivity exclusively for NS1 antigen for diagnosis of dengue. (Table 1)

In this study, $7.92 \%$ cases were positive for IgM only and $10.23 \%$ cases were positive for both NS1 antigen and IgM antibodies. This clearly concludes that we would have missed a few positive cases if only NS1 antigen or only $\operatorname{IgM}$ antibodies were detected. Similarly, various studies also report a significant increase in detection of dengue when both the assays were performed together in a single sample (Schilling et al., 2004 and Ampaiwan et al., 2008) (Table 1). Our findings are also similar to a study done by Fauziah Md et al. which found that on 208 dengue suspected fever cases, NS1 antigen was positive in 67 patients $(32.2 \%)$ and a total of 107 patients (51.4\%) were positive for $\operatorname{IgM}$ and $\mathrm{IgG}$ antibodies while a combination of these tests would raise the detection of dengue fever in 129 cases out of 208 patients $(62 \%)$.

Table.1 Detection Rate of MAC-ELISA and NS1 Assay in Dengue Positive Samples of Group I ( $\mathrm{n}=391)$

\begin{tabular}{|c|c|c|c|}
\hline & \multicolumn{2}{|c|}{ NS1 antigen ELISA } & \multirow[t]{2}{*}{ Total } \\
\hline & Negative & Positive & \\
\hline \multirow{2}{*}{$\begin{array}{l}\text { MAC ELISA } \\
\text { Negative }\end{array}$} & $198(50.63 \%)$ & $122(31.20 \%)$ & $320(81.84 \%)$ \\
\hline & $31(7.92 \%)$ & $40(10.23 \%)$ & $71(18.15 \%)$ \\
\hline Total & $229(58.56 \%)$ & $162(41.43 \%)$ & $391(100 \%)$ \\
\hline
\end{tabular}

Table.2 Positivity of NS1 antigen and IgM in Early (1-7 days) and Convalescent (8-14 days) phases

\begin{tabular}{|l|l|l|}
\hline Day post onset of illness & NS1 & IgM \\
\hline 1 to 7 days & $157 / 162(96.91 \%)$ & $5 / 71(7.04 \%)$ \\
\hline 8 to 14 days & $5 / 162(3.08 \%)$ & $66 / 71(92.95 \%)$ \\
\hline
\end{tabular}


Fig.1 Showing total no. of suspected Dengue cases at different age group \& gender.

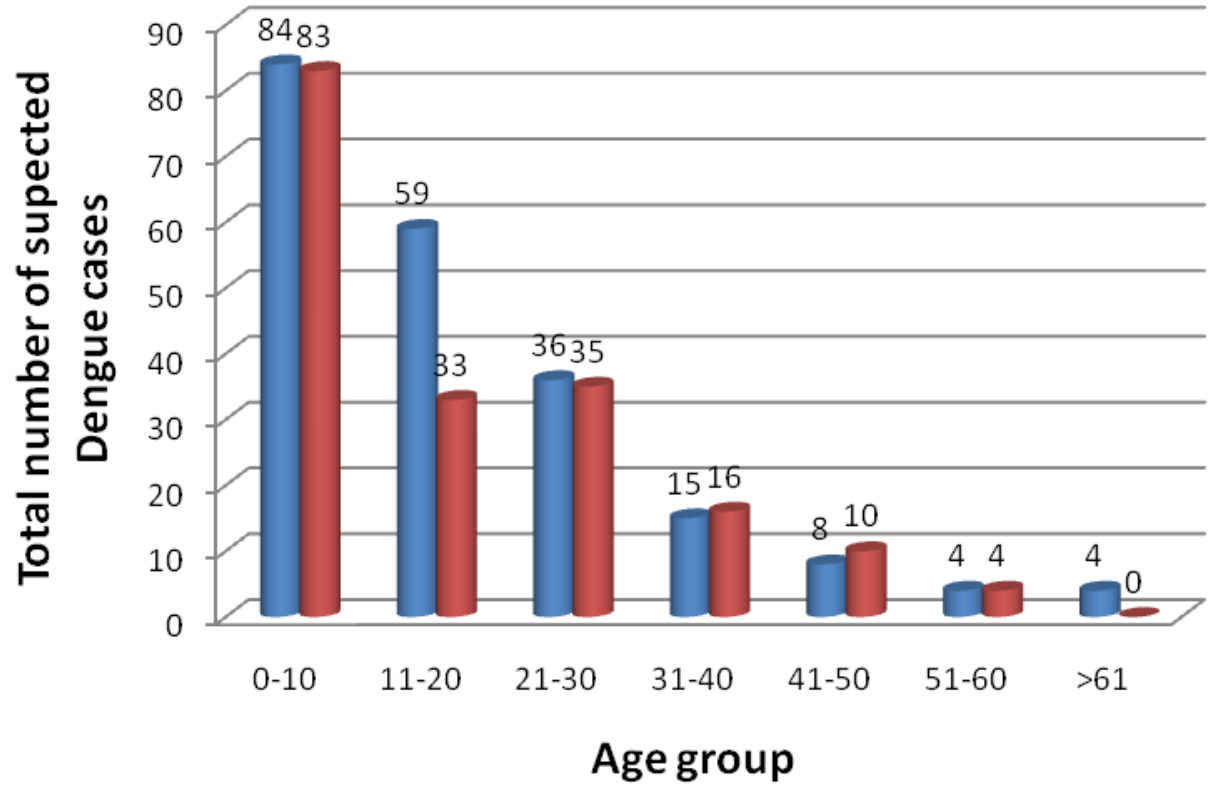

- Male

- Female

Fig.2 Showing total no. of Dengue negative cases at different age group \& gender.

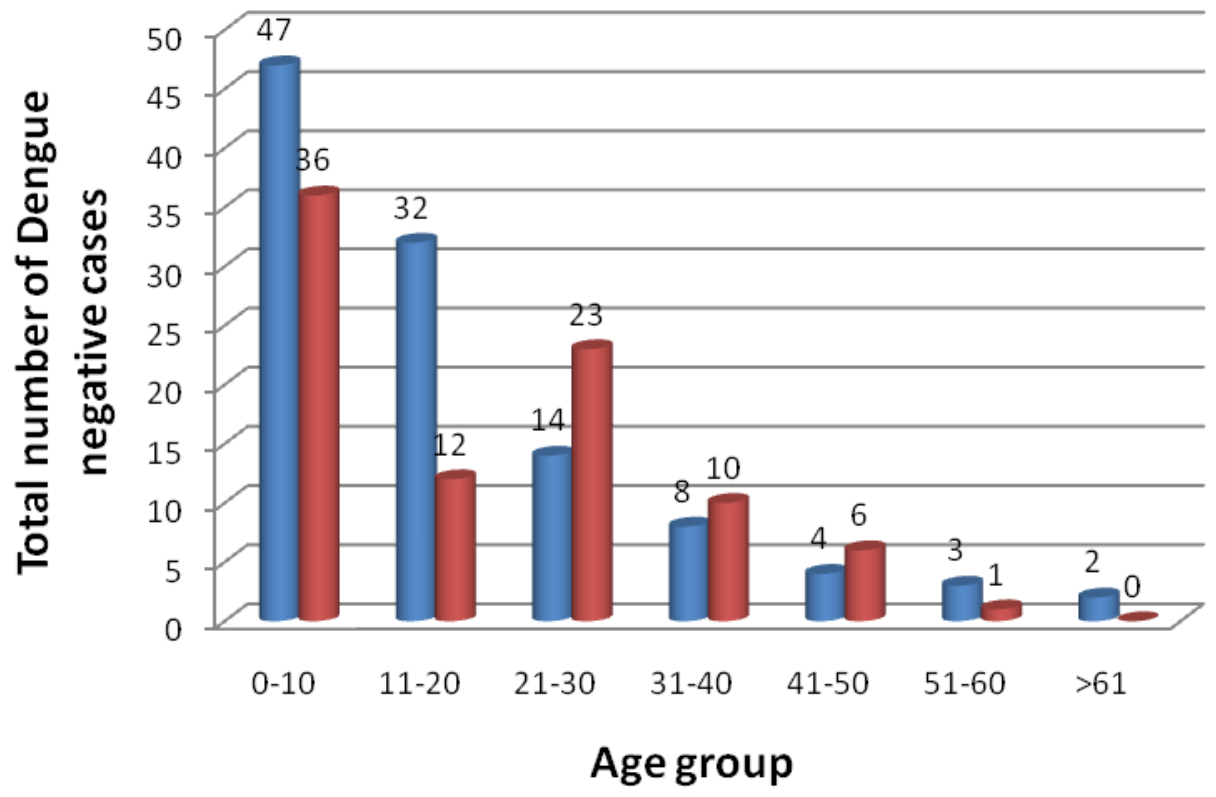

Male

- Female 
Fig.3 Showing total no. of IgM positive \& NS1 positive cases at different age group \& gender

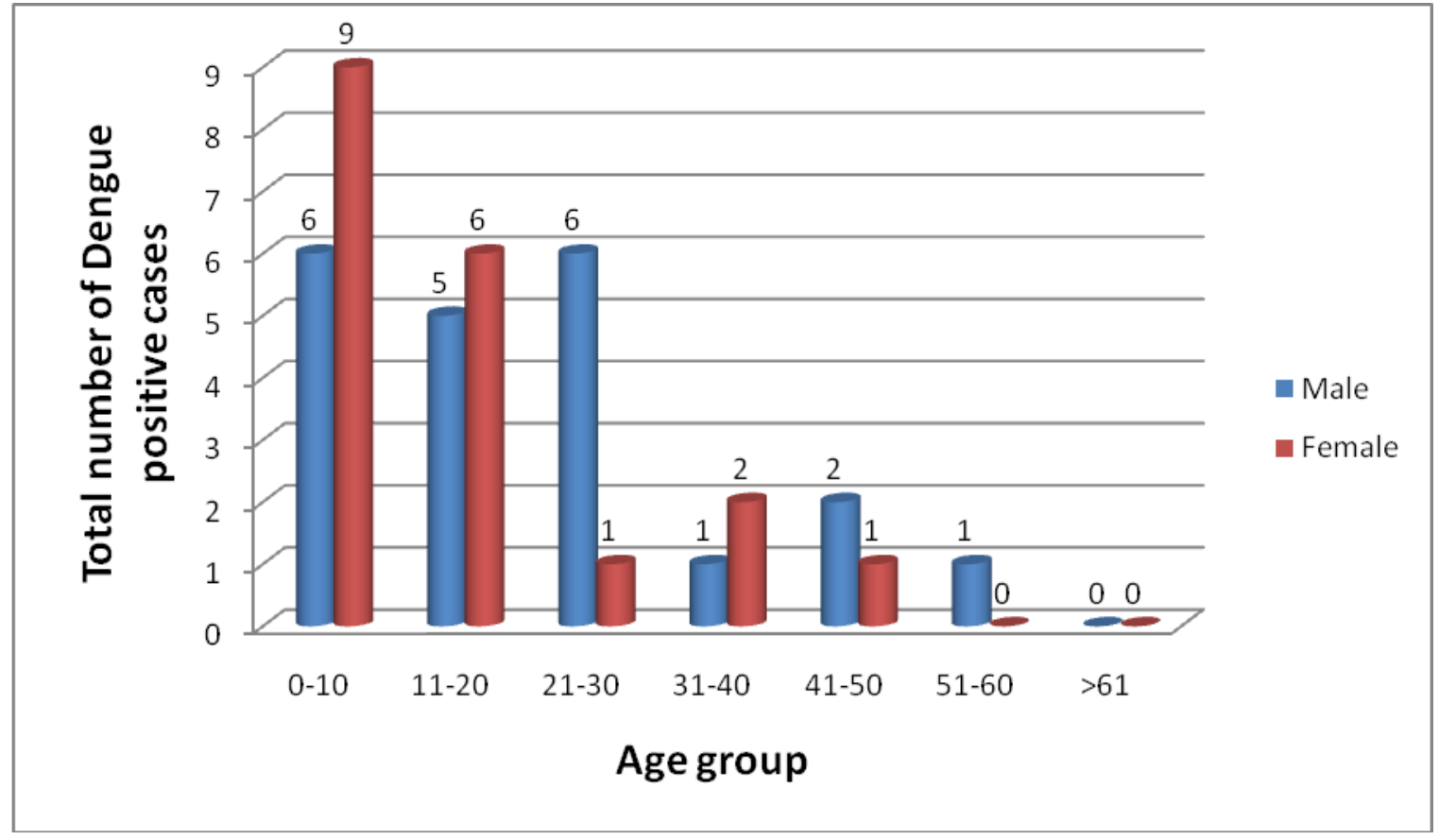

Fig.4 Showing total no. of IgM positive \& NS1 negative cases at different age group \& gender

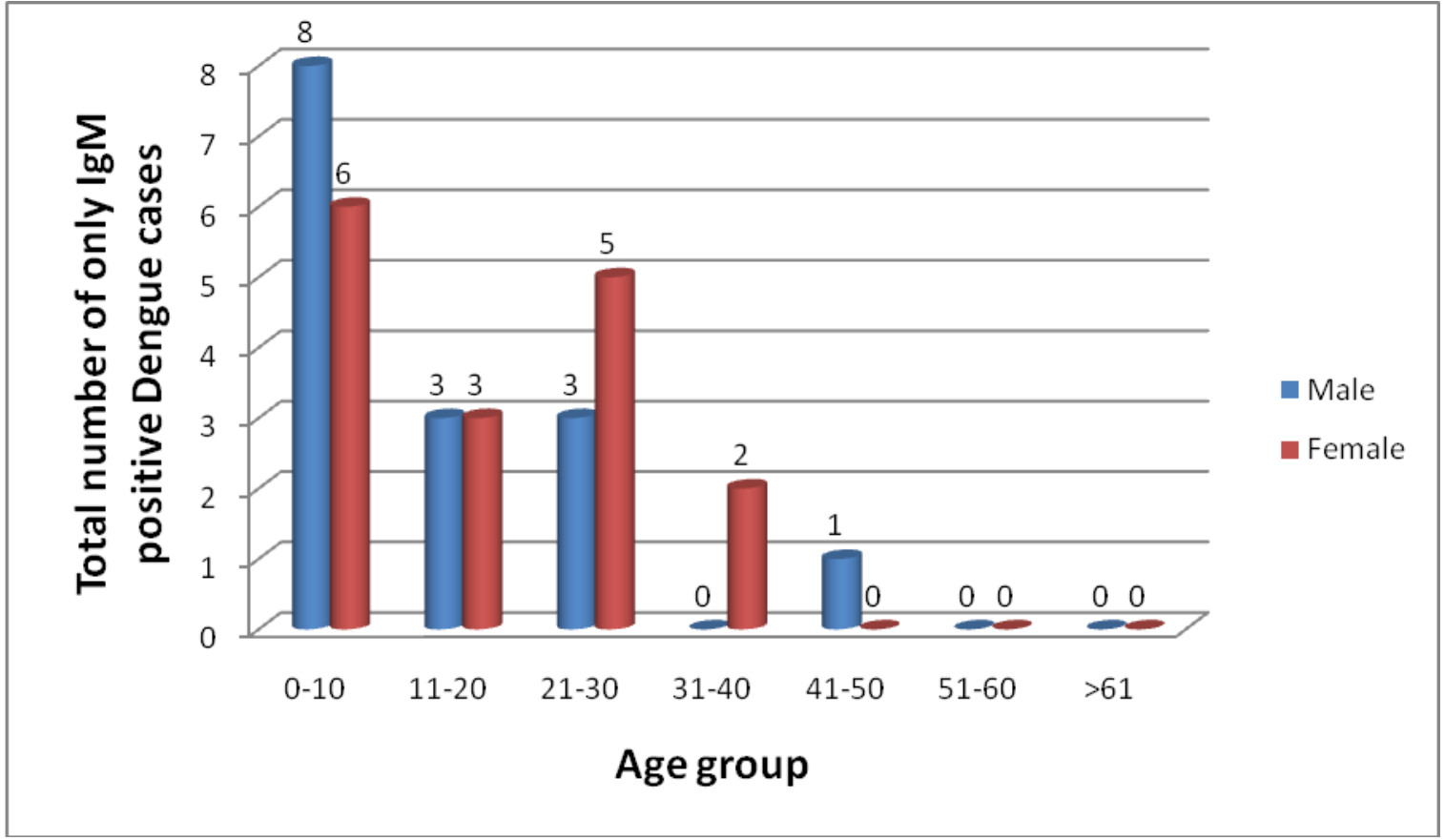

Therefore the dengue NS1 antigen test can be used to complement the current antibody detection tests and the combination of these serological tests would increase the diagnosis efficiency of early diagnosis of dengue illness (Kassim et al.,2011) (Table 2). 
In the present study, NS1 antigen was found to be present from day 2 till day 7 of illness and IgM was found from day 3 of the illness. According to a study by Alcon et al in 2002, NS1 antigen could be detected from day 1 till day 9 of illness (Alcon et al., 2002). Wang et al in 2010 shows NS1 antigen may be detected upto day 14 of illness and IgM will be found from day 3 of illness (Wang et al., 2010) (Table 2). Studies by Kumaraswamy et al., 2007, showed that dengue NS1 Ag ELISA to be more sensitive for diagnosis in the acute phase of primary infection than the secondary infection (Kumaraswamy et al., 2007) (Table 2).

In our study, the highest number of cases belonged to the younger age group and males clearly outnumbered females which was in concordance with Gupta et al., and Chakravarti et al., study which also showed maximum cases in age group of 21-30 years and male prepordance (Gupta et al., 2006 and Chakravarti et al., 2005). The young adults getting more affected reflects the presence of non-immune adult population falling prey to the circulating serotype of dengue virus and involvement in outdoor activities making them more exposed to mosquito bite. Contrasting results were reported by Madhulika et al., 2013 with highest prevalence in females (Madhulika et al., 2013) (Table.3, 4, 5, 6, 7).

In conclusion, our finding suggest that the NS1 antigen assay is very useful and specific tool for the diagnosis of acute dengue infection. However, the sensitivity of the NS1 assay is depended on the level of viremia and host humoral immune response Therefore, combined use of NS1 antigen with dengue IgM test could significantly improve diagnostic sensitivity of dengue infection. Hence by doing both NS1 antigen detection test and $\operatorname{IgM}$ antibody detection test, we can diagnose dengue fever early so that the morbidity and mortality can be reduced. We also conclude that NS1 Ag is an effective tool for the diagnosis of DENV infection, especially with in first 7 days of illness.

\section{Acknowledgement}

I would like to express my profound gratitude to all the participants for their cooperation and for their immense faith they reposed in me.

Conflict of Interest: None to declare.

\section{References}

Alcon, S., Talarmin, A., Debruyne, M. Falconar, A. Deubel, V. and Flamand, M. 2002. Enzyme linked immunosorbant assay specific to Dengue virus type 1 non structural protein NS1 reveals circulation of the antigen in the blood during the acute phase of disease in patients experiencing primary or secondary infection. J. Clin. Microbiol., 40(2): 376-81.

Ampaiwan, C., C. Wathanee, P. Viroj, T. Kanchana, L. Sarapee and Sutee, Y. 2008. The use of Nonstructural protein 1 antigen for the early diagnosis during the febrile stage in patients with dengue infection. J. Paed. Infect. Dis., 27:43-8.

Andries, A.C., V. Duong, C. Nagan, S. Ong, R. Huy, Sroin, K.K. et al. 2012. Field evaluation and impact on clinical management of a rapid diagnostic kit that detects dengue NS1, IgM and IgG. PLoS Negl. Trop. Dis., 6(12): e1993.

Bessof, K., M. Delorey, W.Sun and Hunsperger, E. 2008. Comparison of two commercially available dengue virus (DENV) NS1 capture enzyme- 
linked immunosorbent assays using a single clinical sample for diagnosis of acute DENV infection. Clin. Vaccine Immunol., 15: 1513-8.

Cecilia, D., M.B. Kakade, A.B. Bhagat et al. 2011. Detection of dengue-4 virus in Pune, Western India after an absence of 30 years - its association with two severe cases,\| Virol. J., 8(46).

Chakravarti, A., R. Kumaria. 2005. -Ecoepidemiological analysis of dengue infection during an Outbreak of dengue fever, India,\| Virol. J., vol. 2, article 32.

Datta, S., and Wattal, C. 2010. Dengue NS1 antigen detection: A useful tool in early diagnosis of dengue virus infection. Indian J. Med. Microbiol., 28:107 10 .

Dussart, P., B. Labeau, G. Lagathu, P. Louis, M.R.T. Nunes, Rodrigues, S.G, et al. 2006. Evaluation of an Enzyme Immunoassay for detection of dengue virus NS 1 antigen in human serum. Clin. Vaccine Immunol., 13: 1185-90.

Gubler, D.J., and Meltzer M. 1999. Impact of dengue/dengue hemorrhagic fever on the developing world. Adv. Virus Res., 53: 35-70.

Gupta, E., L. Dar, G. Kapoor, S. Broor. 2006. The changing epidemiology of dengue in Delhi, India. Virol. J. 3(92).

Halstead, S.B. 2007. Dengue. Lancet, 370: $1644-52$

Kassim, F.M., M.N. Izati, Y.M. Apandi and Saat, Z. 2011. Use of dengue NS1 antigen for early diagnosis of dengue virus infection. Southeast Asian J. Trip. Med. Public Health, 42(3): 5629.

Kulkarni, R.D., S.S Patil, G.S. Ajantha, A.K. Upadhya, A.S. Kalabhavi, Shubhada, R.M, et al. 2011. Association of platelet count and serological markers of dengue infection importance of NS1antigen. Indian J. Med. Microbiol., 29: 359-62. Kumaraswamy, V., Wahab, A.H., Chau, S K., Hassan, Z., Chem, YK., Mohammad, M. et al. 2007. Evaluations of a commercial dengue NS1 antigen capture ELISA for laboratory diagnosis of acute dengue virus infection. J. Virol. Methods, 140(2): 75-9.

Madhulika, Mistry, Yogesh, Goswami, Rajesh, K., Chudasama, Dhara, Thakkar. 2015. Epidemiological and demographic characteristics of dengue disease at a tertiary care centre In Saurashtra region during the year 2013. J. Vector Borne Dis., 52: 299303.

Sathish, N., T.S. Vijayakumar, P. Abraham and Sridharan, G. 2003. Dengue fever: its laboratory diagnosis, with special emphasis on $\operatorname{IgM}$ detection. Dengue Bull., 27: 116-21.

Schilling, S., D. Ludolfs, V.A. Le and Schmitz, H. 2004. Laboratory diagnosis of Primary and secondary dengue infections. J. Clin. Virol., 31: 179-84.

Tathe, S., V.V. Chincholkar, D.M. Kulkarni, S.L. Nilekar, R.S. Ovhal and Halgarkar, C.S. 2013. A study of NS1 antigen and platelet count for early diagnosis of dengue infection. Int. $J$. Curr. Microbiol. App. Sci., 2(12): 4044.

Tricou, V., H.T. Vu, N.V. Quynh, C. Nguyen, H.T. Tran, Farrar, J. et al. 2010.Comparison of two dengue NS1 rapid tests for sensitivity, specificity and relationship to viraemia and antibody responses. BMC Infect. Dis., 10: 142 .

Vaughn, D.W., S. Green, S. Kalayanarooj et al., 2000. Dengue viremiaiter, antibody response Pattern, and virus serotype correlate with disease 
severity,\| J. Infect. Dis., vol. 181: no.1, pp. $2-9$.

Wang, S.M., and Sekaran, S.D. 2010. Evaluation of commercial SD Dengue Virus NS1 antigen Capture Enzyme Linked Immunosorbent Assay Kit for early diagnosis of Dengue Virus Infection. J. Clin. Microbiol., 48(8): 2793-7

WHO. 2009. Dengue Guidelines for diagnosis, treatment, prevention and control.

\section{How to cite this article:}

Rajani Ranganath and Basavaraj V. Peerapur. 2016. Evaluation of NS1 Antigen Detection for Early Diagnosis of Dengue Virus Infection in a Tertiary Care Hospital in Karnataka, India. Int.J.Curr.Microbiol.App.Sci. 5(12): 710-717. doi: http://dx.doi.org/10.20546/ijcmas.2016.512.081 